\title{
Prevention and control strategies of overweight and obesity
}

\section{Background information}

Overweight and obesity are leading nutrition and life style related disorders of clinical and public health concern. Overweight and obesity are generally defined as abnormal or excessive fat accumulation ${ }^{1}$ and weight gain that exceeds the standard indicator values. ${ }^{2}$ Body Mass Index (BMI) is the most commonly used measure to define overweight and obesity. It is calculated as the ratio of a person's body weight to the square of his body height $\left(\mathrm{kg} / \mathrm{m}^{2}\right){ }^{1,3}$ The BMI is no direct measure of body fat as it cannot distinguish between body fat and muscle mass. ${ }^{1,4}$ However, research has shown that BMI indicates a high correlation with direct measurements used to determine body fat ${ }^{3,5,6}$ and BMI provides the most useful population-level measure of overweight and obesity as it is the same for both sexes and for all ages of adults. ${ }^{1}$ A high BMI can therefore act as an indicator of a high level of body fat. According to the World Health Organization WHO classification system, adults with a BMI between $25 \mathrm{~kg} / \mathrm{m}^{2}$ and under $30 \mathrm{~kg} / \mathrm{m}^{2}$ are taken as overweight and a BMI of $30 \mathrm{~kg} / \mathrm{m}^{2}$ or more as obese. ${ }^{1}$

For under-five children overweight and obesity are measured by the WHO child growth standards. Overweight is weight-for-height greater than 2 standard deviations above WHO Child Growth Standards median; and obesity is weight-for-height greater than 3 standard deviations above the WHO Child Growth Standards median. ${ }^{1}$ For children aged between 5 - 19years, overweight and obesity are defined based on the WHO growth reference for this age group. ${ }^{1}$ Accordingly, overweight is BMI-for-age greater than 1 standard deviation above the WHO Growth Reference median; and obesity is greater than 2 standard deviations above the WHO Growth Reference median. ${ }^{1}$

\section{Causes of overweight and obesity}

The causes of overweight and obesity are very complex, but there are many suggested contributors, such as poor diet and consumption of fast food, increased eating frequency, increased portion sizes, a decrease in physical activity, and energy imbalance between calories consumed and calories expended..$^{1,7-9}$

\section{Health consequences}

Overweight and obesity are an increasing problems worldwide and increase the risk for many diseases such as diabetes, ${ }^{10-12}$ cardiovascular diseases, ${ }^{10,11,13}$ some types of cancer (including endometrial, breast, ovarian, prostate, liver, gallbladder, kidney, and colon), ${ }^{1,10,11}$ and musculoskeletal disorders (especially osteoarthritis - a highly disabling degenerative disease of the joints). ${ }^{1}$ Overweight and obesity are also associated with a higher risk of premature deaths. ${ }^{14,15}$ Overweight and obesity associated co-morbidities are major challenges to the health system and present an important public health problems all over the world. ${ }^{3}$

\section{Prevention and control strategies}

Overweight and obesity, as well as their related non-communicable diseases, are largely preventable. Supportive environments and communities are fundamental in shaping people's choices.
Volume 7 Issue 4 - 2017

\author{
Zemichael Gizaw \\ Public Health Specialist, University of Gondar, Ethiopia
}

Correspondence: Zemichael Gizaw, Public Health Specialist, University of Gondar, Ethiopia, Email ZemichaelI2@gmail.com

Received: October 25, 2017 | Published: November 0I, 2017

The prevention and control options individual or community, organizational and environment based as explained below.

\section{Individual or community based interventions}

People can prevent overweight and obesity by reducing the intake of high energy dense foods (i.e foods high in fat/sugar); increasing the intake of low energy-dense foods(especially vegetables, fruits, legumes, whole grains and nuts); reducing the consumption of sugar-sweetened soft drinks and juices; reducing the level of food prepared outside of the home; reducing portion sizes; ${ }^{1,16}$ increasing energy expenditure like regular physical activity (60minutes a day for children and 150 minutes spread through the week for adults) $)^{1,16,17}$ or increasing participation in active recreation or increasing use of active transport and reducing time spent in sedentary behaviors (especially TV watching); ${ }^{16}$ and reducing the likelihood of the development of a disease or disorder by practicing good life styles. ${ }^{18}$ Population-wide policies and initiatives, such as marketing restrictions on unhealthy foods and nonalcoholic beverages, nutrition labeling, food taxes and subsidies, physical activity policies, and social marketing campaigns are useful to compact overweight and obesity. ${ }^{18,19}$ Moreover all these prevention options have to be integrated with the community or public health system to promote health and health behaviors to the long run. $9,16,19,20$

The community or population based overweight and obesity intervention approaches can be upstream, midstream, or downstream, but most interventions are likely to be upstream and midstream. ${ }^{21}$ The upstream or socio-ecological approach aims to shape the underlying determinants of overweight and obesity. Actions target the food environments, physical activity environments and the broader socioeconomic environments (including taxation, employment, education, housing and welfare), thus indirectly influencing population behaviors. Sectors targeted include all aspects of the food system (such as agriculture, food processing, food distribution, marketing, retail and food service sectors) and sectors that influence the physical activity environment (such as infrastructure, transport, and education sectors). ${ }^{21}$ The midstream or behavioral approaches aim to improve population dietary and physical activity behavior patterns. Midstream approaches will typically be targeted at the settings level, where programs, social marketing, education, and other initiatives to motivate individuals to change diet and physical activity behaviors can be implemented. These settings may include childcare centers, schools, community and recreational facilities, or household 
settings..$^{21}$ The downstream approaches are typically directed towards supporting health services and medical (clinical) interventions. The downstream approaches are typically individual based, rather than population-based. ${ }^{21}$

\section{Organization based interventions}

The healthcare systems need greater emphasis on helping people to stay healthy through stronger investment in prevention, early detection and appropriate interventions to keep people in the best possible health. ${ }^{18}$ The healthcare systems should promote healthy life styles through primary healthcare services by designing effective public health approaches. ${ }^{9,20,22}$ Schools are also responsible to prevent overweight and obesity by promoting physical exercise, health diet and other healthy behaviors. ${ }^{23}$ Moreover, schools should provide more healthful food options to the students if they have a feeding program. ${ }^{24}$ The food industry can play a significant role in preventing overweight and obesity by reducing the fat, sugar and salt content of processed foods; ensuring that healthy and nutritious choices are available and affordable to all consumers; restricting marketing of foods high in sugars, salt and fats, especially those foods aimed at children and teenagers; and ensuring the availability of healthy food choices; providing information (for example, product and menu labeling and responsible marketing; the placement of healthy products in more prominent positions in supermarkets); introducing point of sale menu labeling (i.e. where menu boards contain nutritional and energy content information) and supporting regular physical activity in the workplace. ${ }^{1,18}$

\section{Environment based interventions}

The built environment including the working environment should play roles promote health and healthy behaviors. The built environment can promote healthy life style if the walking network (footpaths and trails, etc.), the cycling network (roads and cycle paths), public open spaces (parks) and recreation facilities (recreation centers, etc.) are well designed. ${ }^{18,22}$ The physical, social, political and economic environments have a profound effect on the way people live and behave. Each day people interact with a wide range of services, systems and pressures in settings such as schools, the workplace, home, and commercial settings. In turn, these settings are influenced by laws, policies, economic imperatives and the attitudes of governments, industry and society as a whole. Each of the features of this complex system has the capacity to inhibit or encourage appropriate dietary and physical activity patterns. ${ }^{16}$

\section{Conclusion}

In general, simple and effective strategies are available to prevent and control overweight and obesity. Governments have a responsibility to coordinate health reform to deliver preventative programs and to make sure adequate supports are put in place to enable individuals, families and communities and the health system to make useful contributions. Addressing overweight and obesity requires much greater multi-sectoral collaboration, leadership and coordination. Furthermore, integrating of overweight and obesity interventions with community or public health program is vital.

\section{Acknowledgements}

None.

\section{Conflicts of interest}

The author declares no conflict of interest.

\section{References}

1. Obesity and overweight. World Health Organization (WHO); 2017.

2. Robert J Kuczmarski, Katherine M Flegal. Criteria for definition of overweight in transition: background and recommendations for the United States. Am J Clin Nutr. 2000;72:1074-1081.

3. Anja Schienkiewitz, Gert BM Mensink, Ronny Kuhnert, et al. Overweight and obesity among adults in Germany. Journal of Health Monitoring. 2017;2(2):20.

4. Must A, SE Anderson. Body mass index in children and adolescents: considerations for population-based applications. Int $J$ Obes. 2006;30(4):590-594.

5. Willett WC. Anthropometric measures and body composition. Nutritional Epidemiology. $2^{\text {nd }}$ ed. USA: Oxford University Press; 1998. p. 244-272.

6. Pietrobelli A, Faith MS, Allison DB, et al. Body mass index as a measure of adiposity among children and adolescents: a validation study. $J$ Pediatr. 1998;132(2):204-210.

7. Duval K, Strychar I, Cyr MJ, et al. Physical activity is a confounding factor of the relation between eating frequency and body composition. Am J Clin Nutr. 2008;88(5):1200-1205.

8. Michael J Devlin, Susan Z Yanovski, G Terence Wilson. Obesity: What Mental Health Professionals Need to Know. Am J Psychiatry. 2000;157(6):854-866.

9. Blair SN, Nichaman MZ. The Public Health Problem of Increasing Prevalence Rates of Obesity and What Should Be Done About It. Mayo Clin Proc. 2002;77(2):109-113.

10. Ng M, Fleming T, Robinson M, et al. Global, regional, and national prevalence of overweight and obesity in children and adults during 1980-2013:a systematic analysis for the Global Burden of Disease Study. Lancet. 2014;384(9945):766-781.

11. Hurt RT, Kulisek C, Buchanan LA, et al. The obesity epidemic: challenges, health initiatives, and implications for gastroenterologists. Gastroenterol Hepatol (NY). 2010;6(12):780-792.

12. Abdullah A, Peeters A, de Courten M, et al. The magnitude of association between overweight and obesity and the risk of diabetes: a meta-analysis of prospective cohort studies. Diabetes Res Clin Pract. 2010;89(3):309319.

13. Guh DP, Zhang W, Bansback N, et al. The incidence of co-morbidities related to obesity and overweight: a systematic review and metaanalysis. BMC Public Health. 2009;9:88.

14. Carmienke S, Freitag MH, Pischon T, et al. General and abdominal obesity parameters and their combination in relation to mortality: a systematic review and meta-regression analysis. Eur J Clin Nutr. 2013;67(6):573-585.

15. Flegal KM, Kit BK, Orpana H, et al. Association of all-cause mortality with overweight and obesity using standard body mass index categories: a systematic review and meta-analysis. JAMA. 2013;309(1):71-82.

16. Best options for promoting healthy weight and preventing weight gain in NSW.

17. John M Jakicic, Amy D Otto. Treatment and Prevention of Obesity: What is the Role of Exercise? Nutr. 2006;64(2 Pt 2):57S -61S.

18. Obesity prevention.

19. World Health Organization (WHO). Population-based approaches to childhood obesity prevention. 2012.

20. Ruth SM Chan, Jean Woo. Prevention of Overweight and Obesity: How Effective is the Current Public Health Approach. Int J Environ Res Public Health. 2010;7:765-783. 
21. Sacks G, Swinburn B, Lawrence M. Obesity Policy Action framework and analysis grids for a comprehensive policy approach to reducing obesity. Obesity Reviews. 2009;10(1):76-86.

22. Michaela Vine, Margaret B Hargreaves, Ronette R Briefel, et al Expanding the Role of Primary Care in the Prevention and Treatmen of Childhood Obesity: A Review of Clinicand Community-Based Recommendations and Interventions. Journal of Obesity. 2013;2013:17.
23. Mahshid Dehghan, Noori Akhtar-Danesh, Anwar T Merchant. Childhood obesity, prevalence and prevention. Nutrition Journal. 2005;4:24.

24. Mary Story, Karen M Kaphingst, Simone French. The Role of Schools in Obesity Prevention. The future of children. 2006;16(1):109-142. 